\title{
Research on the Development of Baseball Pitching Machine Controlling Pitch Type using Neural Network*
}

\author{
Shinobu SAKAI ${ }^{* *}$, Juhachi ODA ${ }^{* * *}$, Shigeru YONEMURA ${ }^{* * * *}$, \\ Kengo KAWATA $^{* * * *}$, Saburo HORIKAWA ${ }^{* * * * *}$ and Hiroyuki YAMAMOTO ${ }^{* * * * *}$ \\ **Department of Human and Mechanical Systems Engineering, Kanazawa University, \\ Kakuma-machi, Kanazawa, Ishikawa, 920-1192, Japan \\ E-mail: sakai@t.kanazawa-u.ac.jp \\ ***Department of Human and Mechanical Systems Engineering, Kanazawa University, \\ Kakuma-machi, Kanazawa, Ishikawa, 920-1192, Japan \\ E-mail: oda18@t.kanazawa-u.ac.jp \\ ****PFU Co., Ltd., \\ Nu 98-2 Unoke, Kahoku, Ishikawa, 929-1192, Japan \\ *****Kinkicresco Co., Ltd., \\ 3-3-12 Jyounan, Ikeda, Osaka, 563-0025, Japan
}

\begin{abstract}
The most common commercial pitching machines for baseball are the "arm" type and the "two rollers" type. These machines tend to have certain limitations. In particular, it is very difficult to simultaneously change both ball speed and direction. In addition, some types of pitches, such as the curveball or screwball, are not easily achieved. In this study, we will explain the hardware and software design of a new "intelligent" pitching machine which can pitch repeatedly with selectable speed, direction and ball rotation. The machine has three rollers and the motion of each is independently controlled by a hierarchical neural network. If the ball speed, direction and rotation are given as input data to this network, signals for controlling the three rollers are produced as output data. The results of a throw experiment with the machine that we developed are shown, which has the ability to pitch assorted breaking balls with a wide range of speeds, from 19.4 to $44.4 \mathrm{~m} / \mathrm{s}$. The machine has a speed error of less than about $3 \%$, and a distance error of about $0.15 \mathrm{~m}$ (twice the length of a ball's diameter).
\end{abstract}

Key words: Baseball, Pitching Machine, Neural Network, Intelligent Machine, Learning

\section{Introduction}

Pitching machine is a machine that throws a ball instead of a pitcher in batting practices, and it is widely used in venues ranging from professional baseball to batting centers. The most important purpose of a pitching machine is to reproduce the throws of an adversary pitcher, which will be useful for the improving batting techniques. The most common commercial pitching machines for baseball are the "arm" type and the "two rollers" type. Some pitching machines can pitch a high-speed ball (fastball) and a breaking ball, but these machines have certain limitations. In particular, it is very difficult to change ball speed and direction simultaneously. In addition, it is difficult to simulate some breaking balls, such as a curveball or a screwball.

The machine used in this study has three rollers and two actuators; the motion of

${ }^{*}$ Received 6 Feb., 2007 (No. T2-04-0629) Japanese Original : Trans. Jpn. Soc. Mech. Eng., Vol. 71, No. 702, C (2005), pp.555-560 (Received 28 May, 2004) [DOI: 10.1299/jsdd.1.682] 


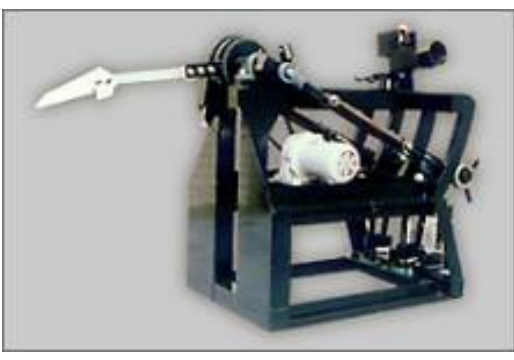

Figure 1 Pitching machine (arm type)

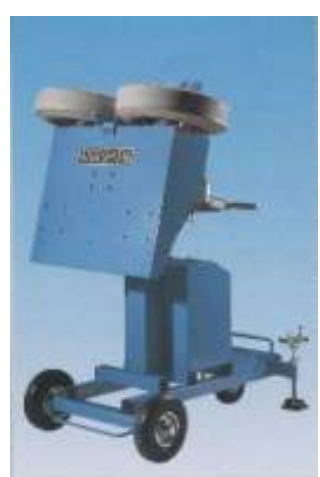

Figure 2 Pitching machine (two rollers type)

each is controlled independently by a hierarchical neural network (NN). If the ball speed, direction and rotation are given to this machine as input data, detailed motion information about the three rollers and the projected angles is produced as output data. With this mechanism comes the production of a new pitching machine that can pitch balls repeatedly in the way the batter desires, controlling both ball speed and pitch type. Here, the system of a pitching machine with the NN and its performance evaluation are described.

\section{The present condition of pitching machines and their problems}

One important function of a pitching machine for baseball has traditionally been to throw a ball at a very high speed. The most common commercial pitching machines for baseball are the "arm" type and the "two rollers" type. The principal mechanism of the arm type machine is a spring and crank lever that imitates a human arm when pitching a ball (see Fig. 1). The two rollers type is shown in Fig. 2. This machine uses two rollers to project a ball by taking advantage of the frictional force between the rollers and the ball. Generally, the ball speed can be controlled with this type of machine, but direction changes are very difficult or impossible to achieve. With the two rollers machine type, the spin direction given to a ball is controllable only on the plane in which the roller is turning, because a ball flies only on that plane. This can change the ball spin and ball speed by changing the number of the ball's turns on its plane. However, the plane must be spun manually in order to produce arbitrary breaking balls, which requires a decision about the axis to be based on trial and error under the given conditions.

If we wish to develop a high fidelity pitching machine for baseball capable of throwing a wide range of pitches with full freedom and control over both the speed and angular velocity of the ball, it is important that both of these quantities be independent of one another ${ }^{(1),(2)}$. Thus, the authors decided to develop a machine with the important functions (throwing freely and producing the breaking balls, speed and direction a batter desires) that are currently missing from commercial pitching machines. For example, the machine produces several types of pitches. The machine detects its surrounding wind or temperature conditions and adjusts the characteristics of its pitches accordingly in order to produce pitches with the desired course and speed. Such a machine is called an "intelligent" pitching machine.

\section{Overview of a new type of pitching machine}

A new type of pitching machine that can voluntarily control two parameters affecting the direction and speed of the ball has already been developed by the authors ${ }^{(3)}$. This machine consists of three rollers arranged around the circumference of a ball in its discharge position, and the rotary direction of the ball can be controlled over a full 360 degrees as the 


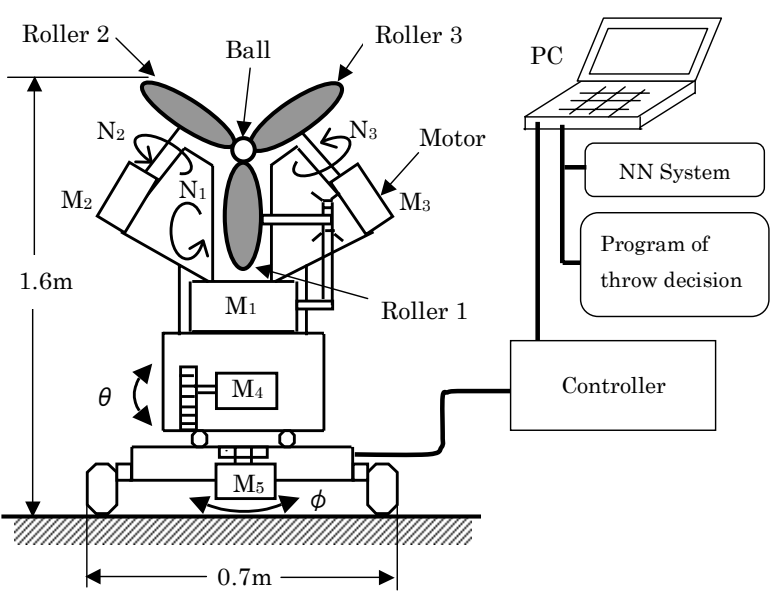

Figure 3 Constitution of the new pitching machine

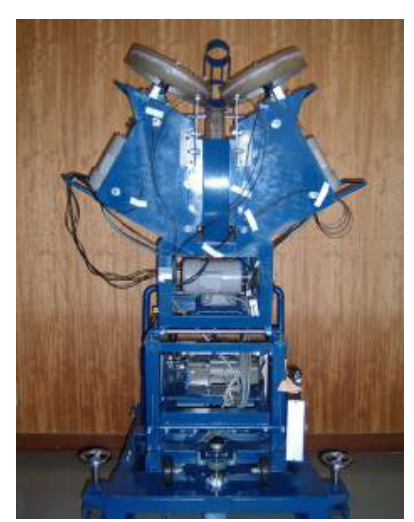

Figure 4 New pitching machine

(three rollers type)

three rollers create three planes on a three-dimensional axis by variously changing the turn frequency of each roller. With this structure, various kinds of throws with variable pitch types and speeds became possible. Before the development of this machine, only the direction and speed of pitched balls were being considered. However, the needs of this field were investigated, and control over the speed and spin for each pitched ball was the most important feature desired for these machines. It is obvious that choice of direction was the next most important feature. In this study, the new "intelligent" pitching machine, which can be programmed to control pitches based on three parameters (speed, pitch type and direction), will be developed.

The structure of the new type of pitching machine that was developed by the authors is shown in Fig. 3, and a photograph of the machine is shown in Fig. 4. This machine employs three rollers, which includes one more roller than the pitching machines typically found today. A baseball is thrown with frictional force by the rubber tire, and this roller is installed around the circumference of a ball in its discharge position at $120^{\circ}$ intervals. Motors are installed in each respective roller with a link through a V-belt, in which the number of revolutions can be adjusted from 0 to $2300 \mathrm{~min}^{-1}$, and these motors can be controlled independently. Additionally, the machine has $\mathrm{M}_{4}$ and $\mathrm{M}_{5}$ motors in its lower section, which are necessary for it to be able to throw various kinds of balls in arbitrary directions. That is, this machine has a mechanism that can change the vertical angle $\theta$ from $-5^{\circ}$ to $5^{\circ}$ and the horizontal angle $\phi$ of the machine itself from $-6^{\circ}$ to $6^{\circ}$, as shown in Fig. 3. With the new type of pitching machine, which adopted these new mechanisms, a wide range of speeds (up to $44.4 \mathrm{~m} / \mathrm{s}$ ), pitch types (fastball, curveball or screwball) and directions, can be pitched, as desired. Additionally, each motor is connected to a personal computer (PC) through a controller, and the number of revolutions of each of the motors can be controlled by the PC. In addition, this machine is equipped with various sensors that measure the number of revolutions $N_{1}, N_{2}$ and $N_{3}$ of three rollers, the vertical angle $\theta$, the horizontal angle $\phi$ and the initial velocity $V$ of the ball.

\section{The throw control method with the neural network}

A hierarchical neural network $(\mathrm{NN})$ that includes an optimization technique is used to control the intelligent pitching machine, whose many functions are explained in Chapter 3. Next, we describe this control method. Using this control system, if five units (horizontal coordinate $X$ and vertical coordinate $Y$ of the direction position, initial velocity $V$ and pitch type parameters $B_{X}$ and $B_{Y}$, to be mentioned later) are given as inputs, five units (number of revolutions $N_{1}, N_{2}$ and $N_{3}$ of three rollers, vertical angle $\theta$ and horizontal angle $\phi$ ) will be 


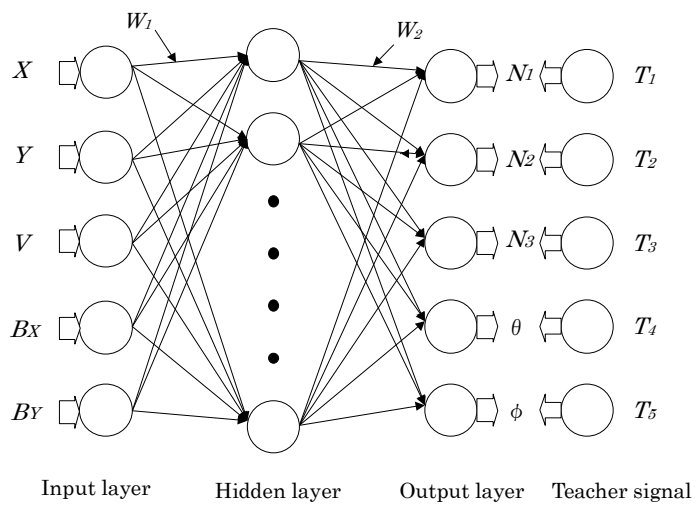

Figure 5 Neural network model

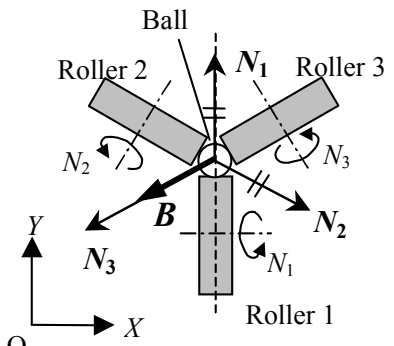

(a)

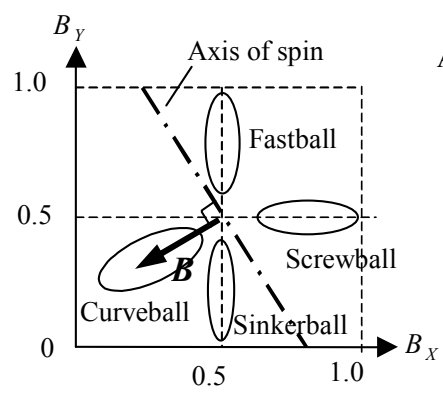

(b)

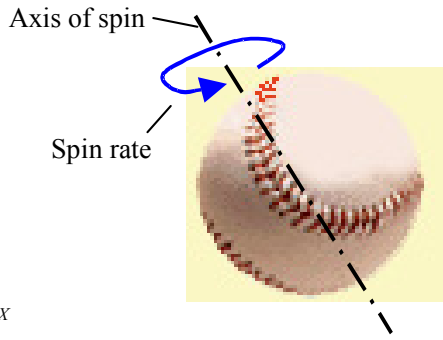

(c)

Figure 6 Pitch type parameters: $B_{X}, B_{Y}$

given by the system as outputs to enable a throw, that will produce the pitch that the batter desires. At first, a different method of calculating the trajectory of the ball theoretically was considered. The technique is described in a paper ${ }^{(1)}$ previously discussed by the authors. It calculates the ball's trajectory by measuring the aerodynamic forces and Magnus effect of a ball ${ }^{(4)}$. However, it requires a lot of trouble and time, and its control accuracy is not high.

Thus, the hierarchy type $\mathrm{NN}^{(5)}$, which can learn the relationship between these inputs and outputs without needing this complicated theory, was adopted in this study. Some teaching data is given, the $\mathrm{NN}$ itself learns, and it is thereby possible to obtain the correct outputs. For complicated problems, such as when the wind causes slight changes in the trajectory of a ball, the NN method offers a very effective solution. Figure 5 shows the neural network model used in this study. From this figure, we can see that this NN model has a hierarchy network structure with three layers, whose input layer is formed by five variables $\left(X, Y, V, B_{X}\right.$ and $\left.B_{Y}\right)$, and whose output layers are formed by five variables $\left(N_{1}, N_{2}\right.$, $N_{3}, \theta$ and $\phi$ ). We gave teaching data $T_{i}$ to this $\mathrm{NN}$ model, which it learned by decreasing the sum of square errors $\left(=\Sigma\left(T_{i}-N_{i}\right)^{2} / 2\right)$ between outputs $N_{i}$ and $T_{i}$ using the back propagation algorithm, and the $\mathrm{NN}$ was built with the exact outputs provided by this method.

\section{Pitch type parameters: $B_{X}, B_{Y}$}

The authors suggest a technique which incorporates two pitch type parameters ( $B_{X}$ and $\left.B_{Y}\right)$. Now, the number of turns $N_{1}, N_{2}$ and $N_{3}$ of the three rollers in the newly developed pitching machine changes, and it is assumed that these numbers are related by $N_{3}>N_{1}=N_{2}$. When the angular momentum given to the ball by each roller is then expressed in a vector, these vectors $N_{1}, N_{2}$ and $N_{3}$, corresponding to each roller, which are shown in Fig. 6(a) at intervals of $120^{\circ}$. In this case, vector $N_{3}$, produced by roller 3 , is the biggest, and vectors $N_{1}$ 


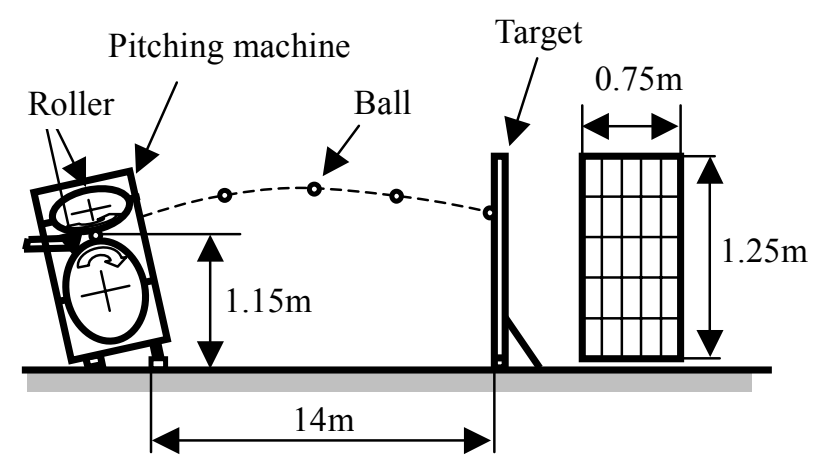

Figure 7 Experimental conditions

and $N_{2}$, produced by rollers $N_{1}$ and $N_{2}$, are equal to each other. When these three vectors are added $\left(\boldsymbol{N}_{\mathbf{1}}+\boldsymbol{N}_{\mathbf{2}}+\boldsymbol{N}_{3}\right)$, a vector $\boldsymbol{B}$ is given, as shown in Fig. 6(a). The size of vector $\boldsymbol{B}(|\boldsymbol{B}|)$ is divided by the number of maximum turns ( $\left|\boldsymbol{N}_{\max }\right|$ ) of the roller. Vector $\boldsymbol{B}$ is mapped onto the 2-dimensional coordinate of horizontal $B_{X}$ and vertical axis $B_{Y}$ at a center position $B_{X}$ $=B_{Y}=0.5$, as shown in Fig. 6(b). The length of the defined vector $\boldsymbol{B}$ shows the ball's rotation number (spin rate). The line is perpendicular to the direction of vector $\boldsymbol{B}$, expressing an axis centered by the ball's spin.

For example, in the case of $\boldsymbol{B}$, the spin axis and rate of a ball are shown in Fig. 6(c), and this type of pitch can be described as a curveball. The same display can pitch types, such as a fastball, screwball and sinkerball, as a range, as shown in Fig. 6(b). In the range of $0<B_{Y}$ $<0.5$, the ball spin type is a top spin. In the range of $0.5<B_{Y}<1.0$, the ball spin type is a back spin. In the case of $B_{X}=B_{Y}=0.5$, there is no spin (knuckleball). In general, if the two pitch type parameters $B_{X}$ and $B_{Y}$ are used, all types of pitch can be expressed, because a specific pitch type is characterized by its spin axis and rate. The propriety of this technique is examined by a basic performance examination in the next chapter.

\section{Performance evaluation of the new intelligent pitching machine}

\subsection{Basic performance examination}

First, we examined the pitch types produced when we changed the number of revolutions $N_{1}, N_{2}$ and $N_{3}$ of the three rollers and the vertical $\theta$ and horizontal $\phi$ angles of this pitching machine. The ball used for the experiments was the same hard baseball as that used for professional baseball. All experiments were conducted in the same room, and the target (height: $1.25 \mathrm{~m}$, width: $0.75 \mathrm{~m}$ ) was set $14 \mathrm{~m}$ away from the pitching machine, as shown in Fig. 7. The throw experiments were conducted in order to choose an optional combination of number of roller revolutions and angles $\left(N_{1}, N_{2}, N_{3}, \theta\right.$ and $\left.\phi\right)$ with the machine. Figure 8 shows the resulting position coordinates of the pitch targets from 115 different pitch patterns tested. All of the experimental data reflects the average value of three different pitches thrown for a single pattern. The data confirms that most domains can be targeted, as shown in the figure. Figure 9 shows a relationship between the numerical sum of the number of revolutions of the three rollers $\left(N_{1}+N_{2}+N_{3}\right)$ and the ball speed $V$. The results obtained in this experiment showed a linear correlation between the two variables, and also that this machine can pitch a range of ball speeds, from 19.4 up to $44.4 \mathrm{~m} / \mathrm{s}$.

In these experiments, the state of the ball's spin in each type of pitch was monitored using a high-speed video camera. Figure 10 shows one example of the results, reflecting a combination of $N_{1}=2100 \mathrm{~min}^{-1}, N_{2}=N_{3}=1500 \mathrm{~min}^{-1}$, and $\theta=\phi=0^{\circ}\left(B_{X}=0.5, B_{Y}=0.6\right)$, which produces a fastball (straightball). The pitching machine throws a ball with a clear back spin, which is almost the same as a ball thrown by an actual pitcher. Also, the location 


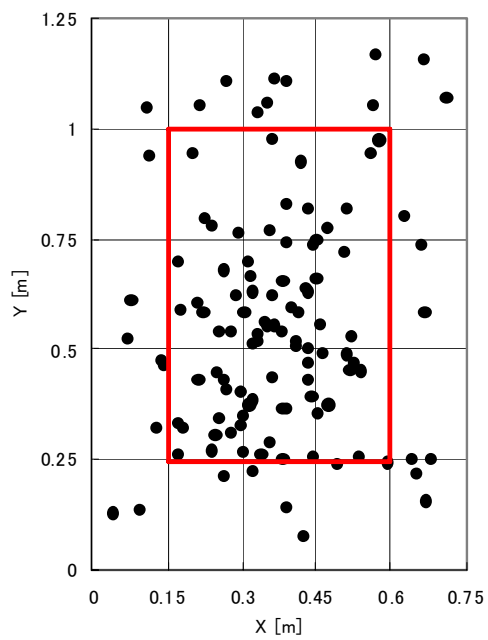

Figure 8 Example of pitching ball

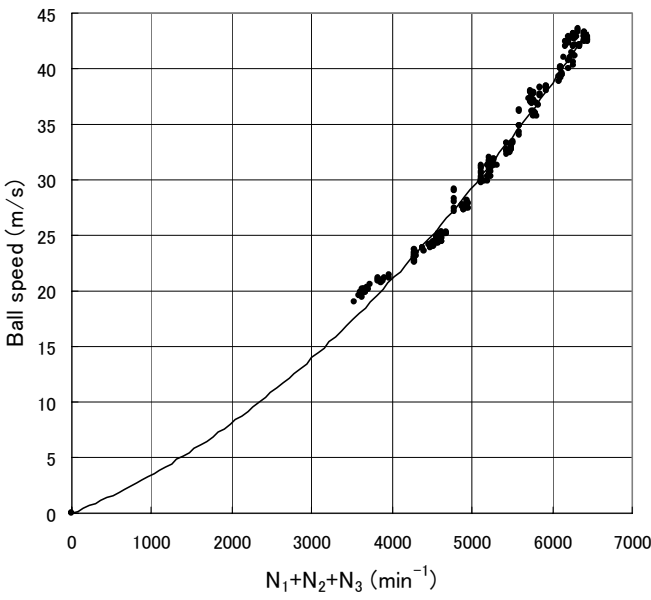

Figure 9 Relation between ball speed and

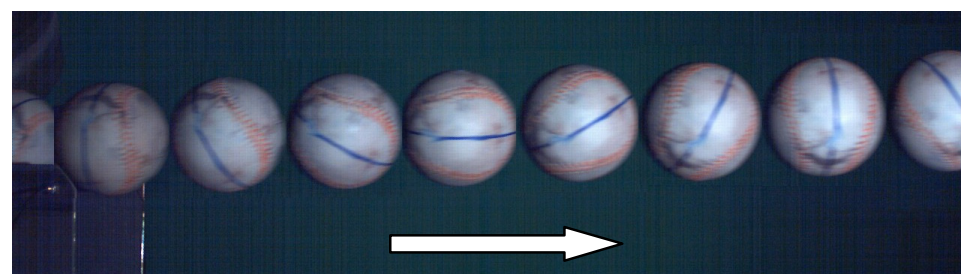

Figure 10 Behavior of the spin of a fastball using a high-speed video camera (3ms/frame)

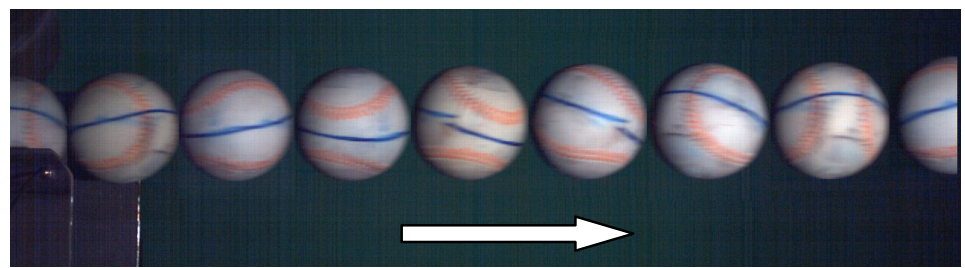

Figure 11 Behavior of the spin of a curveball using a high-speed video camera (3ms/frame)

$\left(B_{X}=0.4, B_{Y}=0.42\right)$ of a curveball is shown in Fig. 11. The ball exhibits follow spin, and the rotation axis of the ball's spin is slightly inclined from a vertical line. If we compare these results to those in Fig. 6(c), the axis direction and ball spin rate are clear for both balls. In addition, though the other pitches' spin types (screwball, sinkerball and knuckleball) are not shown in the figure, they have been confirmed using the image captured by the high-speed video camera. It is said that because this technique can create various kinds of pitches by pitch type parameters $B_{X}$ and $B_{Y}$ (explained in the beginning of the chapter), is it a sufficiently appropriate method.

\subsection{Total performance evaluation}

The NN was made to learn teaching data using the back propagation algorithm as experimental data in Section 6.1. One example of a learning process is shown in Fig. 12. The results show that, regardless of the amount of teaching data, the number of errors decreases with learning frequency. We examined an actual pitch produced by an NN whose learning process had been completed. We tested the NN model's production of given pitch types, which were learned from the teaching data about the 115 pitches, as shown in Fig. 8. 


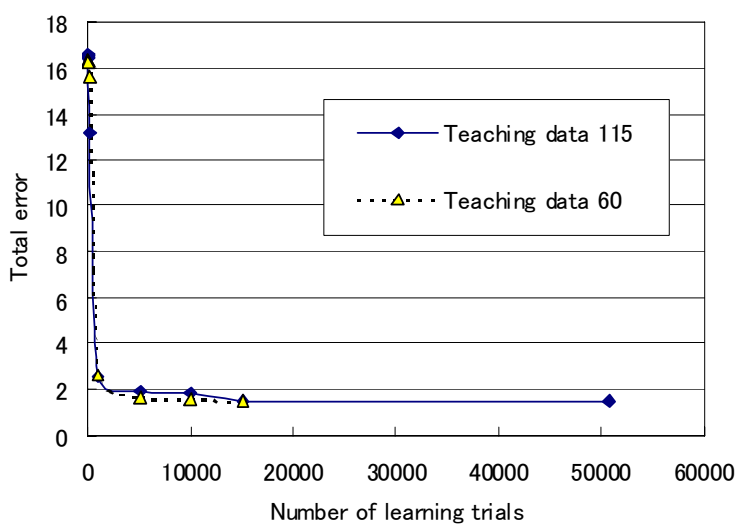

Figure 12 Error convergence situation

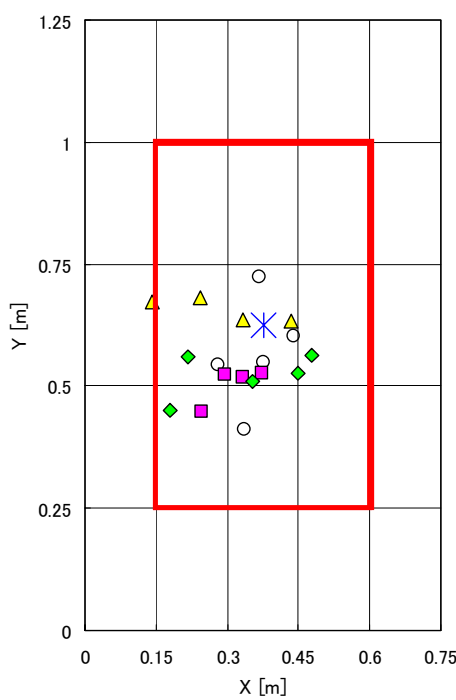

(a) Cases by pitch types

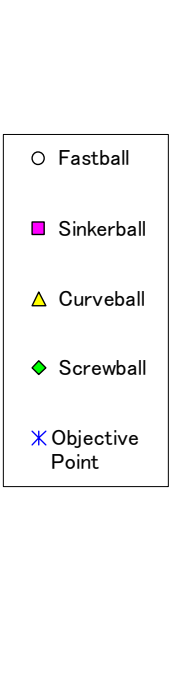

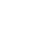

(b) Cases by balt
Figure 13 Experimental results of ball using $\mathrm{NN}$ control

An example of these results is shown in Fig. 13. In other words, a predetermined direction, five speeds $(70,90,110,130$ and $150 \mathrm{~km} / \mathrm{h}$ ), and four pitch types (fastball, sinkerball, curveball and screwball) were chosen, and were produced based on the NN variables $N_{1}, N_{2}, N_{3}, \theta$ and $\phi$. The seal (*) in these figures represents the desired position of a ball (the center of the target). Also, Fig. 13(a) shows throw examination results with a different symbol for each pitch type: fastball $\bigcirc$, sinkerball $\square$, curveball $\triangle$ and screwball $\diamond$. Conversely, each pitched ball's speed is shown as a parameter based on the experimental results in Fig. 13(b).

From these results, we find no clear correlation between the speed and spin type of pitched balls. There is a degree of dispersion on the whole, but the ball is always centered at least partially over the desired position. In addition, these are good results, indicating that the strike zone positioning of the ball would not be altered by either ball speed and pitch type (ball speeds of $150 \mathrm{~km} / \mathrm{h}$ are included in machines of professional baseball grade). The pitching machine we developed performs better than commercial machines, because in a moment its pitches can be diverted to the preferred directions with a wide range of speeds and assorted pitch types, believed to represent all of those currently present in baseball.

The speed error $\Delta V$ and distance error $\Delta r$ were defined in order to evaluate these results more quantitatively than is done above: 


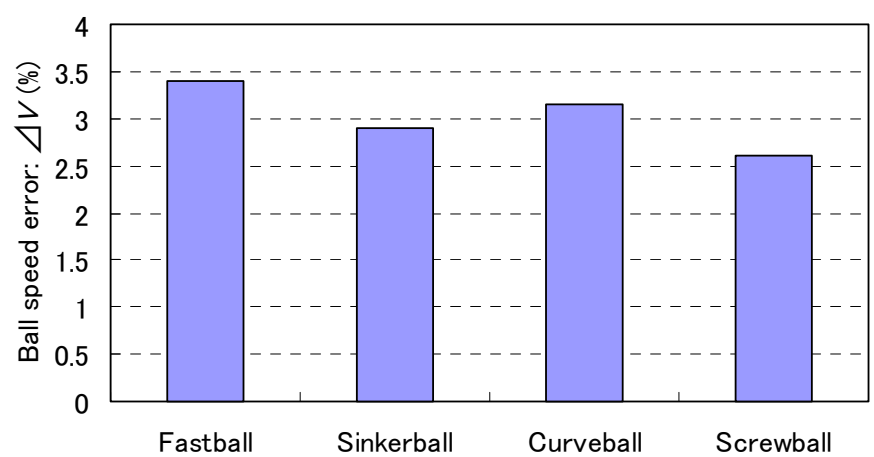

Figure 14 Relation between ball speed error $(\Delta V)$ and pitch type

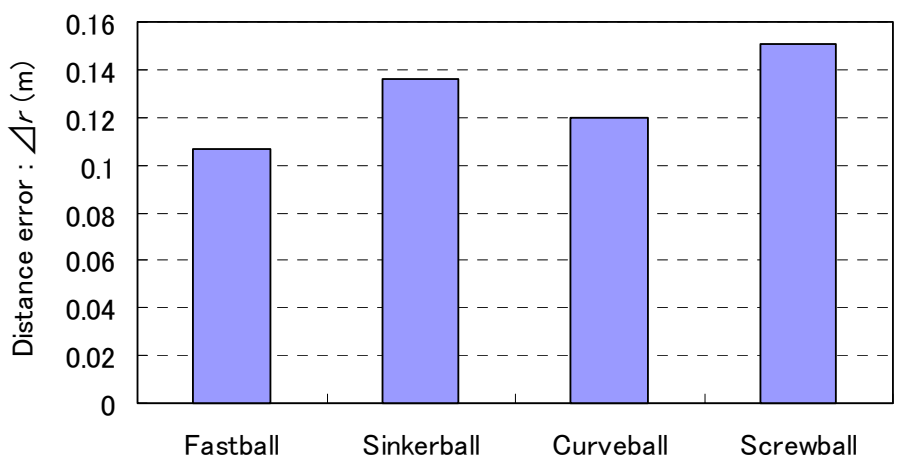

Figure 15 Relation between distance error $(\Delta r)$ and pitch type

$$
\Delta V=\left|\frac{V^{\prime}-V}{V}\right| \times 100
$$

where $V$ is the objective value of ball speed and $V^{\prime}$ is the experimental value of ball speed.

$$
\Delta r=\sqrt{\left(X^{\prime}-X\right)^{2}+\left(Y^{\prime}-Y\right)^{2}}
$$

where $X$ is the objective value of the $\mathrm{x}$-coordinate, $X^{\prime}$ is the experimental value of the $\mathrm{x}$-coordinate, $Y$ is the objective value of the $\mathrm{y}$-coordinate and $Y^{\prime}$ is the experimental value of the y-coordinate.

Figure 14 shows calculations of the preferred speed and speed error $\Delta V$ for a throw to produce all four pitch types (fastball, sinkerball, curveball and screwball). For every pitch type, the difference in this value was the result of variation, and was small, ranging between $2.6 \%$ and $3.4 \%$. For this reason, the machine exhibited an almost linear relationship between the numerical sum of the number of revolutions of the three rollers $\left(N_{1}+N_{2}+N_{3}\right)$ and the ball speed $V$, as shown in Fig. 9, because learning this characteristic using the NN was easy. Figure 15 shows a calculation of the directional error $\Delta r$ of each pitch for four different pitch types (fastball, sinkerball, curveball and screwball).

As seen in Fig. 15, as in $\Delta V$, the directional error $\Delta r$ varied by the pitch type to the extent that its values ranged from about $0.11 \mathrm{~m}$ (in the case of a fastball) to $0.15 \mathrm{~m}$. In this experiment, the diameter of each baseball was about $0.072 \mathrm{~m}$, so the error $\Delta r$ is no more than the size of two balls; that is, this machine has been constructed with very high accuracy and the errors produced are small. For these reasons, as in the case of ball speed, there are many possible combinations of the number of revolutions among the three rollers. Additionally, a real baseball ball has a seam and is a not perfectly spherical in shape. The effect of the seam is that depending on its position, contact with the ball can vary for any 
given turn frequency of the rubber roller. It is believed that the friction factor between the rubber roller and a ball with a seam changes depending on the ball's loading position ${ }^{(6),(7)}$.

\section{Conclusion}

In this study, we describe the design of a new type of baseball pitching machine that uses three rollers. The machine determines the number of roller rotations and the necessary angles of a pitch using a neural network, and as a result, it can pitch a ball with the desired direction, speed and spin. The results show that this machine has the ability to pitch assorted pitch types with a great range of speeds, from 19.4 to $44.4 \mathrm{~m} / \mathrm{s}$. The throw accuracy of this machine is almost complete with regard to speed and spin. Regarding the accuracy of direction, the prediction variables had an error maximum of about $0.15 \mathrm{~m}$ (twice the length of a ball's diameter). That is enough accuracy to far surpass other present pitching machines used today. It may be said that this is a new and intelligent pitching machine with higher practicality ${ }^{(2)}$.

Finally, great assistance was given to this study by Mr. Takumi KAWAMURA and Keisuke TAKAKUWA, who were seniors at this university during while it was being conducted. For their help in the production and testing of the machine, the authors wish to express great thanks. Additionally, concerning the new type of pitching machine described here, including the structure and control method, Japanese patent application (No. 2001-45941) has already been filed at the Japan Patent Office.

\section{References}

(1) Oda, J., Sakai, S., Yonemura, S., Kawata, K., Horikawa, S. and Yamamoto, H., Development research of intelligent pitching machine using neural network, Transactions of the Japan Society of Mechanical Engineers, Series C, Vol.69, No.678 (2003), pp.135-140.

(2) Mish, S.P., and Hubbard, M., Design of a degree-of-freedom baseball pitching machine, Sports Engineering, Vol.4, No.3 (2001), pp.123-133.

(3) Oda, J., Sakai, S. and Sakano, K., Industrialization study of a new type intellectual pitching machine with artificial intelligence technology, Kanazawa University 2002 important expense study abstract, (in Japanese),(2003), pp.19-22.

(4) Adair, R.K., (Nakamura, K., Translator), Physics of baseball, Kinokuniya bookstore, (in Japanese), pp.10-36, 1996.

(5) Hiromi Hirano, A neural network to make with C language, Personal media, (in Japanese), (1991), pp.15-46.

(6) Hendee, Sh.P., Greenwald, R.M. and Crisco, J.J., Static and dynamic properties of various baseballs, Journal of Applied Biomechanics, Vol.14, No.4 (1998), pp.390-400.

(7) Nicholls, R.L., Miller, K. and Elliott, B.C., Modeling deformation behavior of the baseball, Journal of Applied Biomechanics, Vol.21, No.1 (2004), pp.9-15. 